\title{
CCXXXII. GLUCOSONE.
}

\author{
BY KENDAL CARTWRIGHT DIXON AND KENNETH HARRISON. \\ From the Biochemical Laboratory, Cambridge.
}

(Received October 17th, 1932.)

IT has been suggested by Hynd [1927] that glucosone is an essential intermediary in carbohydrate metabolism and is partly responsible for the symptoms of insulin hypoglycaemia. The evidence on which these assumptions are based is not very satisfactory, and attempts have therefore been made to detect glucosone in vivo and in vitro under physiological conditions.

\section{EXPERIMENTAL.}

Preparation of glucosone. The original method of Fischer [1889] is lengthy, but a review of other methods by Hynd [1927] provides no good alternative. Evans, Nicoll, Strouse and Waring [1928] describe the production of glucosone by the oxidation of fructose with copper acetate, and a further method is described below.

Riley, Morley and Friend [1932] used selenium dioxide as an oxidising agent in the preparation of substituted glyoxals; they showed that fructose (but not glucose) reduced hot selenious acid, but they did not investigate the products formed.

A solution of $50 \mathrm{~g}$. fructose and $15 \mathrm{~g}$. selenious acid in $200 \mathrm{cc}$. water was heated for 3 hours under reflux on a boiling water-bath, and on cooling filtered from the reddish deposit of selenium. To the filtrate $70 \mathrm{~g}$. lead acetate in 150 cc. water were added, and the solution after cooling in ice was filtered from the precipitated lead selenite. Ice-cold $\mathrm{Ba}(\mathrm{OH})_{2}$ was now added until the solution was strongly alkaline, and the precipitated lead glucosone compound filtered off and washed thoroughly until free from fructose. From this stage the preparation now followed exactly that of Fischer [1889]. From the syrup so obtained (after purification by dissolution in alcohol and evaporation in vacuo) the osazone of glucose was prepared by warming to $37^{\circ}$ with phenylhydrazine acetate for 30 minutes, filtering off and recrystallising from alcohol (see Table I).

Table I. Melting-points.

(a) Glucose phenylosazone from glucose

(b) Glucose phenylosazone from glucosone made by this method

(c) Mixture of (a) and (b) 
Reactions of glucosone. Glucosone gives Schiff's reaction immediately in the cold, and is also bound by sodium sulphite; it therefore probably contains a free aldehyde group. A strong solution gives an immediate precipitate in the cold with 2:4-dinitrophenylhydrazine; the osazone (of glucose) formed crystallises in needles M.P. 252-3 ${ }^{\circ}$ (corr.); Glaser and Zuckermann [1927] give 256-7 " bei raschem Erhitzen." For the detection and estimation of glucosone in dilute solution, an equal volume of the reagent $(0.5 \mathrm{~g}$. 2:4-dinitrophenylhydrazine in $30 \mathrm{cc} .2 \mathrm{~N} \mathrm{HCl}$ [Case and Cook, 1931]) is added, and the solution allowed to stand at $37^{\circ}$ for an hour; further standing is liable to bring down glucose and fructose if these are present. This reaction is delicate, a precipitate being given under these conditions with $0.03 \%$ glucosone solution. Trichloroacetic acid does not interfere.

Glucosone is not appreciably destroyed by evaporation in vacuo provided the solution is acid. A small quantity of glucosone was dissolved in $100 \mathrm{cc}$. blood-serum, and the proteins precipitated with $5 \mathrm{~g}$. trichloroacetic acid. The filtrate was made up to $100 \mathrm{cc}$., and $20 \mathrm{cc}$. were withdrawn (solution A). The remainder was evaporated in vacuo at $45^{\circ}$ to a syrup, and again evaporated after the addition of $50 \mathrm{cc}$. water; the residue was diluted to $80 \mathrm{cc}$. and $20 \mathrm{cc}$. withdrawn (solution B). To solutions A and B $10 \mathrm{cc}$. of the 2:4-dinitrophenylhydrazine reagent were added, and after standing at $37^{\circ}$ for an hour the precipitates were weighed:

$\begin{array}{cccc}\text { Weight of osazone from A } & \ldots & \ldots & 31 \mathrm{mg} \text {. } \\ \text { Weight of osazone from B } & \ldots & \ldots & 29 \mathrm{mg} .\end{array}$

The loss is not greater than the experimental error involved.

Attempted detection of glucosone. In the first series of experiments attempts were made to detect glucosone in the blood of rabbits in hypoglycaemic convulsions following a large overdose of insulin. Details are given in Table II. The insulin was injected intravenously unless otherwise stated.

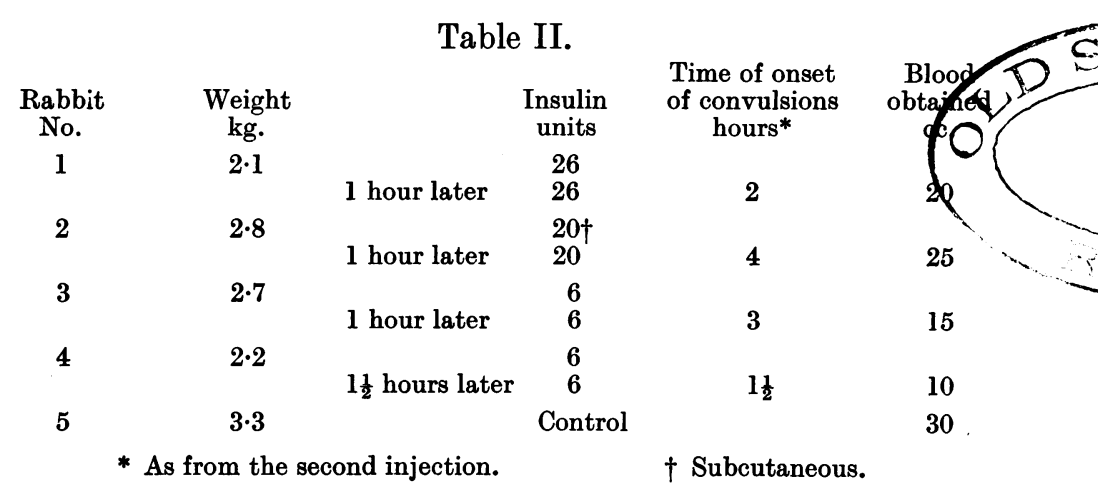

When convulsions occurred the animals were killed by a blow on the head, and rapidly bled from the neck into $10 \mathrm{cc} .100 \%$ (w/v) trichloroacetic acid. The filtrate and washings from the precipitated proteins were evaporated 
in vacuo at $45^{\circ}$ to small bulk, and an equal volume of 2:4-dinitrophenylhydrazine reagent was added. In no case was any precipitate formed after an hour at $37^{\circ}$. In all cases a deep red coloration, more marked in the insulinised animals than in the control, was observed on adding the reagent; this may be due to the presence of glyoxals other than glucosone. Rabbits 1 and 2 were fed on carrots for 24 hours previous to injection; 3 and 4 were starved for 48 hours previously, while the control was fed normally.

Since these experiments gave a negative result, a further attempt was made to detect the formation of glucosone from insulin and fructose in the presence of liver tissue. The technique employed was essentially that of Neuberg and Gottschalk [1924] and Gottschalk [1925] in a similar investigation. The following flasks were set up:

\begin{tabular}{lrrrr} 
& A & B & \multicolumn{1}{c}{ C } & D \\
Liver, g. & 100 & 100 & 100 & 100 \\
Calcium sulphite, g. & 4 & 4 & 4 & 4 \\
Fructose, g. & 2 & 2 & 2 & 2 \\
Insulin, units & 40 & - & 40 & -20 \\
Antiglyoxalase, mg. & - & - & 20 & 20
\end{tabular}

To each flask were added $100 \mathrm{cc}$. phosphate buffer at $p_{\mathrm{H}} 7 \cdot 0$ and $45 \mathrm{mg}$. 8-hydroxyquinoline sulphate (neutralised with $\mathrm{NaOH}$ ) as antiseptic. The flasks were incubated at $37^{\circ}$ for 24 hours, a slow stream of air being passed through to stir the contents and render the system aerobic. After deproteinising with trichloroacetic acid, the filtrate and washings were evaporated to small bulk in vacuo, $50 \mathrm{cc}$. water added and again evaporated; any methylglyoxal or acetaldehyde present was thus removed. The solutions were filtered, diluted to $20 \mathrm{cc}$. and after adding $10 \mathrm{cc}$. of the reagent were allowed to stand at $37^{\circ}$ for an hour. No precipitation occurred.

\section{Discussion.}

From the evidence available it does not seem that glucosone plays any important part in intermediary metabolism. The evidence of Hynd [1927] rests on a superficial similarity between the convulsions of insulin hypoglycaemia and those produced by glucosone: it was also found that adrenaline and pituitrin exerted an antagonising influence in both cases. The injection of other glyoxals, however, also causes convulsions: thus Kermack, Lambie and Slater [1927] found that methylglyoxal, and again [1929] that hydroxypyruvic aldehyde were toxic, though the last observation has been disputed by Hynd [1931]. Moreover, a very large dose of glucosone is required to produce the effect; Herring and Hynd [1928] found that $1.7 \mathrm{mg}$./g. body weight was the convulsant dose for rabbits: calculation shows that the glucosone must be present in the blood (assuming that it is not immediately removed) to the extent of more than $1 \%$. Unless, therefore, glucosone disappears from the blood very rapidly, the failure to detect it in appreciable quantities (more than $0.01 \%$ ) renders it unlikely that the convulsions of insulin hypoglycaemia 
are even partly due to its presence. Evidence that glucosone is not rapidly removed from the blood was found by Hynd [1927]; 15 minutes after the injection of a small quantity of glucosone into a mouse, the apparent bloodsugar (determined by MacLiean's method, which is affected by glucosone) had risen from $0.161 \%$ to $0.238 \%$. Kermack, Lambie and Slater [1929] have pointed out that the absence of convulsions when glucose is injected along with insulin renders it improbable that glucosone causes these convulsions. Further, it is curious that the injection of glucose should alleviate the convulsive condition, since in a system glucose $\stackrel{\text { insulin }}{\longrightarrow}$ glucosone the increase in the concentration of glucose should increase the production of glucosone correspondingly.

The attempted isolation of glucosone from incubated liver tissue also failed. Antiglyoxalase was added to flasks $\mathrm{C}$ and $\mathrm{D}$ to prevent the oxidation of glucosone, if it were formed, to gluconic acid, since experiments indicate that glyoxalase promotes this change. (Further work is in progress on this question, since it is contrary to the finding of Levene and Meyer [1915].) There is thus no evidence that glucosone is formed by the liver, even with added insulin; Clift and Cook [1932, private communication] have shown that glucosone does not occur in fresh liver, and we have confirmed this result. It is useless to attempt to account for the discrepancy which has been pointed out [Holden, 1926 and others] between the reducing power and rotation of blood filtrates by the presence of glucosone, since Groen and Meyer [1932] have shown that reduced glutathione is responsible, even in the case of diabetic patients under insulin treatment. The possibility of glucosone acting as an intermediary cannot be excluded entirely, since Walker [1932] has observed that it is formed from glucose by a mould of the flavusoryzae group of Aspergilli; it has not been found possible, however, to detect it in the animal body.

\section{Summary.}

1. A method is outlined for the preparation of glucosone from fructose by oxidation with selenious acid; a method for its detection is also described.

2. Glucosone does not occur to the extent of more than $0.01 \%$ in the blood of rabbits suffering from convulsions caused by insulin, and it seems that this condition is therefore due to the lowered blood-sugar alone, and not to the accumulation of glucosone.

3. No evidence was obtained that glucosone is formed by the liver, even in the presence of insulin, nor does it occur in fresh liver.

4. There is thus no evidence that glucosone is formed in the intermediary metabolism of the animal body.

We wish to express our thanks to Sir F. G. Hopkins, to Dr Hamilton McCombie and to Dr T. S. Hele for their advice and criticism; and to Mr J. O. Giršavičius and Mr F. P. Clift for a number of suggestions. 


\section{REFERENCES.}

Case and Cook (1931). Biochem. J. 25, 1319.

Evans, Nicoll, Strouse and Waring (1928). J. Amer. Chem. Soc. 50, 2667.

Fischer (1889). Ber. deutsch. chem. Ges. 22, 87.

Glaser and Zuckermann (1927). Z. physiol. Chem. 167, 37.

Gottschalk (1925). Biochem. Z. 155, 348.

Groen and Meyer (1932). Biochem. J. 26, 624.

Herring and Hynd (1928). J. Physiol. 66, 267.

Holden (1926). Biochem. J. 20, 263.

Hynd (1927). Proc. Roy. Soc. Lond. B 101, 244. (1931). Biochem. J. 25, 11.

Kermack, Lambie and Slater (1927). Biochem. J. 21, 40.

- (1929). Biochem. J. 23, 410.

Levene and Meyer (1915). J. Biol. Chem. 22, 337.

Neuberg and Gottschalk (1924). Biochem. Z. 146, 164.

Riley, Morley and Friend (1932). J. Chem. Soc. 1875.

Walker (1932). Nature, 130, 582. 